\title{
Gender and cognitive factors influencing information seeking of graduate students at Kenyatta University Library
}

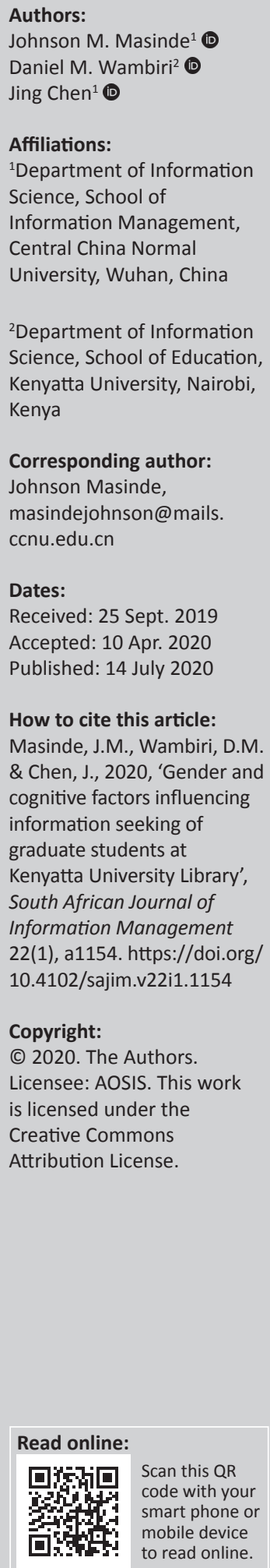

Background: Gender has been identified as a possible influencing factor in users' informationseeking process. Previous studies have alluded to the fact that gender as a variable may be useful for a better understanding of the cognitive and social background of human information processing and may have important implications in the information-seeking process. Although a number of studies have investigated gender, amongst other variables, as having an effect on the information-seeking process of users, no attempt has been made to investigate the relationship between gender and cognitive factors on the information-seeking patterns of graduate students of Kenyatta University Library.

Objective: The study investigates gender and cognitive factors influencing the informationseeking process of graduate students at Kenyatta University Library.

Methodology: To achieve this objective, the study developed a theoretical framework which can be used by academic libraries as a basis for implementing both digital and reference desk services in order to meet the dynamic user needs. The study then investigated whether there were any gender differences through the correlation coefficient in the context of expectancy theory. The motivational process amongst the male and female users was then examined to establish whether there was any difference.

Results: This study found no gender difference in all the variables considered, including interaction service quality, outcome (need satisfaction,) service satisfaction, users' performance of service, past experience, expectancy and effort.

Conclusion: This study found no gender difference in all the variables investigated. The implication of the findings was that there is no need for mainstreaming gender in service programming in the library service.

Keywords: gender; cognitive factors; graduate students; information seeking; academic libraries.

\section{Introduction}

Information-seeking behaviour is broad and involves a set of actions that an individual takes to express their information needs, seek information, evaluate and select information and, finally, use this information to satisfy their information needs (Brindesi, Monopoli \& Kapidakis 2013). It occurs when an individual senses a problem situation which internal knowledge does not satisfy, normally referred to as the mental state 'Anomalous State of Knowledge' (Kassim 2017). Hsieh and $\mathrm{Wu}$ (2015) asserted that information seeking is a natural and necessary mechanism of human existence. It is a basic activity of all people and is manifested through particular behaviours.

At the root of information seeking are personal cognitions which are known as cognitive needs. Savolainen (2013) argued that there are three categories of cognitive needs: need for new information, need to elucidate the information held, and need to confirm the information held. The motives that give rise to cognitive needs, and result in information seeking, are physiological, affective and cognitive (Masinde 2016). Cognitive approaches examine the individual user attributes as the main driving forces behind information-seeking behaviour (Masinde 2016). Such approaches would help libraries to answer basic questions such as, why do students approach, stop or avoid using university libraries. 
Studies have employed the expectancy theory in the investigation of cognitive factors that influence information seeking (Masinde 2016; Savolainen 2013; Wu, Tang \& Tsai 2014). The expectancy theory suggests that people are motivated if valence, instrumentality and expectancy are present. The variable investigated in the context of the above theory is gender. Gender has previously been identified as a variable shaping information-seeking behaviour (Hsieh \& $\mathrm{Wu}$ 2015). Gender refers to the state of being male or female. Studies have revealed multiple physical, mental and social differences in information-seeking behaviour between men and women (Hsieh \& Wu 2015; Savolainen 2013). For instance, Hsieh and $\mathrm{Wu}$ (2015) stated that information seeking may vary according to the gender of the user, making it a variable in the information-seeking process.

The study of gender as a factor influencing human behaviour may therefore be helpful when striving to obtain a better understanding of the cognitive and social bases of human information processing, which may also have significant implications for the designing of information services and systems (Kassim 2017). Gender differences, if any, will therefore be investigated through the correlation coefficient in the context of Vroom's expectancy theory which was improved by Porter and Lawler (1968). This is a cognitive motivational theory and it will be expected to help explain the relation between gender and cognitive factors that influence the information-seeking behaviour of graduate students in using the library services (Porter \& Lawler 1968). The motivational process between men and women will then be examined to establish whether gender influences the information-seeking behaviour of graduate students at the Kenyatta University Post Modern Library. Accordingly, the study will be user-centred, shifting from previous studies which have been system-centred.

\section{Conceptual framework}

The conceptual model in this study is based on the Expectancy Value Theory as propounded by Vroom (1964) and imported by Campbell et al. (1970). The common factors are valence, expectancy, instrumentality, value of outcome, and effort. These perceptions are tempered by the service personnel's (interaction service quality) and users' past experience. These last factors influence service users whilst they are performing the service.

The study assumes an awareness of cultural and biological identities amongst the respondents. It is significant that this research expected to develop a theoretical framework or model which can be used by academic libraries as a basis for developing both digital and reference desk services that would meet dynamic user needs. Accordingly, gender differences, if any, were investigated through the correlation coefficient in the context of the expectancy theory. The motivational process amongst men and women was examined to establish whether there was a difference.

\section{Literature review}

Gender has been identified as one of the variables influencing information seeking (Hsieh \& Wu 2015; Khaola \& Mabilikoane
2015; Savolainen 2013). For instance, Khaola and Mabilikoane (2015), in a study of cancer information service, found that use of the service was mainly by women $(80 \%)$. In another study by Sacks (1994), as quoted by Conrad (2014), it was found that high school student attitudes towards computers and their use varied by gender. Gender was considered as a major predictor of internet use and attitudes. Men seemed to use browsing for enjoyment whilst women tended to use it for work-related purposes. Equally, Rehman (2016) observed that girls and boys differed in their search patterns when using library services to find answers to specific questions. According to the study, boys tend to use horizontal search methods when searching the web in which they iteratively submit searches and then scan the document snippets returned as search results. In contrast, girls tend to employ vertical search methods in which they open and browse the web pages returned by the search engine without reviewing the list or filtering out irrelevant results. In the end, the strategy adopted by most boys tends to provide superior search performance.

$\mathrm{Wu}$ et al. (2014) also established that women tended to experience more difficulties finding information online. They felt less competent and comfortable using the internet. The study, however, found no link between women and low selfefficacy, meaning it is not the gender variable that determines self-efficacy. Whilst this study alluded to the fact that gender studies in general had been done, it tried to ground gender as a variable within the motivational process theory in the context of academic libraries.

Brindesi et al. (2013), whilst investigating the factors that affect international students' information-seeking behaviour, found that female immigrants had higher social-cultural and psychological adaptation scores than male immigrants, but had lower competencies with regard to the use of computerised library resources and technologies. According to Brindesi et al. (2013), most database searching requires extensive computer use and familiarity, which affected their ability. On the other hand, male immigrants seemed more comfortable around information technologies. Therefore, in this area, men were more satisfied and confident and had

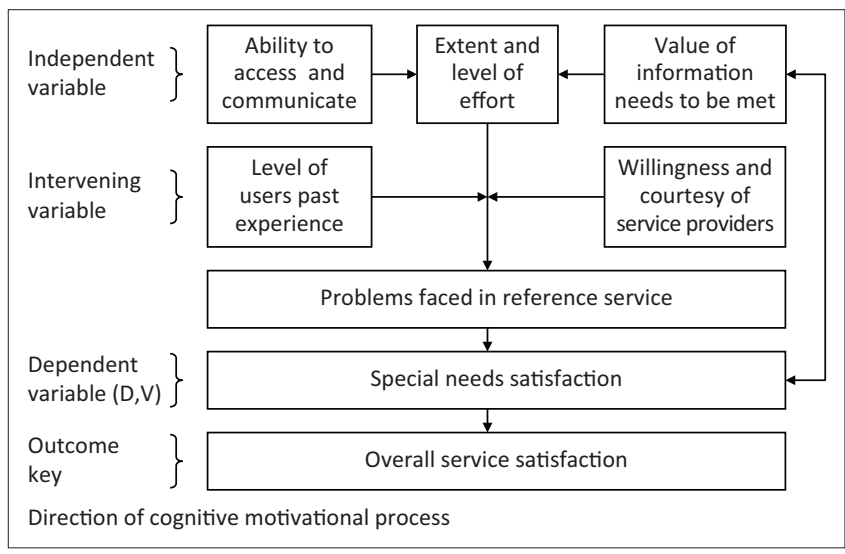

Source: Porter, L.W. \& Lawler, E.E., 1968, Managerial attitudes and performance, Dorsey Press, Illinois.

FIGURE 1: Conceptual framework. 
fewer difficulties than women. Another study by Halder, Ray and Chakrabarty (2010) also argued that gender is an essential variable in information-seeking behaviour. The study stated that satisfaction with a service varied in accordance with the gender of the library user. The men were more comfortable around computers, freely and easily interacting with them, as compared to their female counterparts who seemed to seek the assistance of a reference librarian from time to time.

In summary, the review of literature revealed that the information needs, as well as the information-seeking behaviour amongst men and women, do not show any uniform and consistent pattern. The findings have also revealed that there are variations of needs and behaviour for seeking information between an individual man and woman. In this context, the study seeks to investigate gender and the cognitive factors influencing the information-seeking behaviour of graduate students at Kenyatta University.

\section{Methodology}

The purpose of this study was to investigate gender and the cognitive factors that influence the information-seeking process of graduate students at Kenyatta University. The determinants were based on Vroom's (1964) expectancy theory of motivation. The theory suggests that individuals will be motivated if three factors are present:

- Valence - they must value the outcome;

- Expectancy - they should expect that they are capable of performing the behaviour that is instrumental to the outcome;

- Instrumentality - they must believe that the desired behaviour is instrumental in achieving the valent outcome.

\section{Research design}

According to Nardi (2015), survey research design offers higher representativeness, convenience in collection of data, statistical significance and accuracy in outcomes or findings As a matter of fact, survey research design enables the investigator to gather large amounts of data and employ validated models to arrive at statistically meaningful results (Wisdom \& Creswell 2013). Consequently, this study utilised survey research design as a framework for collection of data. The survey research was cross-sectional and the data were collected using a questionnaire. The data collected were quantitative in nature and were collected from graduate students at Kenyatta University Library. The research then examined the relationship between gender and the cognitive factors that affect the information-seeking process of users.

\section{Variables}

A variable is an attribute on which cases vary. Cases in this study were the students who use the library. The independent variables in this study were the information needs and the expectancy levels. The dependent variable in this study was gender.
Value of outcomes: According to Vroom (1964), value of outcome is the valence. The valence is an individual's subjective judgment about possible outcome or, simply put, the value an individual attaches to rewards. There can be a discrepancy between the anticipated satisfaction from an outcome (valence) and the actual satisfaction. Outcomes are need satisfactions. This variable referred to the anticipated value library attach to their information needs being satisfied through the use of the library service. An outcome is positively valent when a person prefers attaining it to not attaining it, and an outcome has a valence of zero when a person is indifferent to attaining it. This concept assessed differences amongst the male and female users with regard to their subjective judgment about possible outcome. A fivepoint Likert scale was used on each of those aspects.

Effort-to-performance expectancy: This is the second variable in this study. It is the library user's estimated probability that he or she can accomplish a task, given effort or the belief that an individual's effort will lead to realisation of the desired outcomes (Vroom 1964). It is the likelihood that he or she can perform a task at a given level and that his or her effort will lead to successful performance. This was scored on a Likert scale. Two main areas were scored: the conviction that different genders will be able to use the service without problems and that different genders will be able to communicate with the librarians offering the service.

Effort by the user: This is the amount of energy library users exert in the information-seeking process. According to Porter and Lawler (1968), effort is influenced by the value of rewards. The intensity of effort an individual puts in depends on the perception that the effort will result in a desired outcome (Vroom 1964). If the rewards can satisfy an individual's needs for security, self-esteem, autonomy and self-actualisation, the rewards are considered valuable to the individual. Judging the value of rewards, individuals can then determine the effort they put in a search. There is also a perceived probability that there is a close relationship between effort and reward.

Service performance/accomplishment: Library services are produced interactively with the different genders. The study examined how well different genders performed the basic requirement of the reference service without difficulty. This variable focused on how the different genders executed or performed the basic requirements of a reference service in service delivery. Access issues and challenges faced by the different genders whilst executing the tasks were assessed and scored.

User perception interaction service quality: Interpersonal interactions that take place during the service delivery between the reference librarian and the different genders were assessed. The study considered the service provider's response, willingness, courtesy and ability. The different genders' perception of the service quality was assessed. 
User's past experience: This refers to the previous and memorable experiences a user has had with the service - the overall experience by the service user. This was important because it influenced the different genders' own convictions in their own effectiveness (Bandura 1977) or self-efficacy.

Library user service satisfaction: This is the overall assessment of the service experience by the different genders - the subjective judgment of the service by the different genders. It included service features, attribution of service success or failure and perceptions of equity and fairness. Extent of the different genders' satisfaction, the likelihood of repeat use and the possibility of the users recommending the service to somebody else.

\section{Location of the study}

This study was carried out in Kenyatta University Library. The role of a library is to support teaching, learning and research. Kenyatta University was selected because:

- The Post-Modern Library has some of the latest library facilities; inaugurated in 2010 with a sitting capacity of 6000 students. The investment took over 10 million USD (Kenyatta University 2013).

- Kenyatta University has one of the largest number of graduate students (13 193). Accordingly, the library service requires to know how to meet the information needs of such users.

\section{Target population}

The number of registered graduate students stood at 13193 in 2017 (Kenyatta University 2018). This was the target population in this study.

\section{Sampling techniques}

To select the participants, a quota sampling method was utilised. Quota sampling permits investigators to sample a subunit that is of great importance to the investigation. The design demanded that the data be collected at one point in time from a specified population, especially where the data collected was to be subject to correlation analysis (Ary, Jacobs \& Sorensen 2010). The study targeted the collection of data from a sample of 373 out of a total of 13193 postgraduate students. Postgraduate library users were intercepted in the postgraduate floor of the postmodern library. The researcher explained the purpose of the study and then requested them to spare a few minutes to complete the questionnaire. The instruments were collected as soon as they were completed.

\section{Sample size}

Sample size was calculated by determining the sample size of an infinite population and then adjusting it to the sample size of the required population, which was 13193 (Cochrun 1977). For the purpose of this study, a 95\% confidence level was expected. This corresponded to a $Z$ score of 1.96 and a $5 \%$ margin of error (Saunders, Lewis \& Thornhill 2009). Given that the 13193 students had an equal chance of being selected, the sample size was 373 .

\section{Calculation of sample size}

- Sample size was calculated by determining the sample size of the infinite population and then adjusting it to the sample size of the required population (Cochrun 1977)

- Formulae of sample size for infinite population:

- $S=Z^{2} * P^{*}(1-P) / M^{2}$

- where: $S=$ Sample size for the infinite population

- $Z=Z$ score

- $P=$ Population proportion (Assumed to be $50 \%=0.5$ )

- $M=$ Margin of error

- Z score determined based on confidence level

- $\quad$ Confidence level $=95 \%$

- $Z=1.96$

- $M=0.05$

- Substitution of formulae $\left[S=\left(Z^{2}\right) * P^{*}(1-P) /\left(M^{2}\right)\right]$ :

- $S=(1.96)^{2} * 0.5 *(1-0.5) /\left(0.05^{2}\right)$

- $S=3.8416 * 0.25 / 0.0025$

- $S=384.16$

- Adjustment of the sample to the required population:

- Population of Kenyatta University post-graduate students $i$ s $=13193$

- Formula for adjusted sample size $=(S) / 1+[(S-1) /$ Population]

- $\quad$ Adjusted sample size = 384.16/1+ [(384.16-1) / 13193

- Adjusted sample size for Kenyatta University postgraduate students $=373$

\section{Research instruments}

The study used a questionnaire which was developed on the basis of the objectives of the study and the variables. The variables were: Interaction service quality, Effort-toperformance (EP) Expectancy, User needs satisfaction, Service Performance (Service satisfaction), Effort by the user, Outcome, Value of outcomes, User's past experience, and gender relationship with cognitive variables.

\section{Pretesting and/or piloting study}

A pilot study was carried out at a private university, namely, Methodist University, Nairobi campus. It was selected because it has a library that offers digital information services. It is also well funded. The pilot involved seeking permission from the university librarian. Once permission was granted, an appropriate date for distributing the questionnaire was fixed. About 20 participants were selected. The questionnaire was given out to them and collected later. The intention of the pilot study was to validate that the study population would comprehend the questionnaire. The pilot findings revealed that the students comprehended the questions with no difficulty.

\section{Validity}

To collect data, the researcher ensured that the questionnaire was appropriate. This was done by testing internal consistency 
of the questionnaire. Reliability refers to the consistency of a measure of a concept. It entailed asking whether the measure was stable so that the study could be confident that the results relating to that instrument were reliable and did not fluctuate. Cronbach's alpha coefficient was used to test reliability. The figure 0.70 alpha levels was employed as a rule of thumb to denote an acceptable level of reliability.

The instrument already developed for this study was a multiple-item measure for each concept, for example, outcome, EP Expectancy and past experience. This was done to avoid the potential problems associated with overreliance on a single indicative measure. The study used Cronbach's alpha coefficient to test the consistency of the questions. Each variable achieved a coefficient greater than 0.7 .

\section{Data collection techniques}

Questionnaires are known to be easy to construct and administer as they can reach larger populations at relatively cheaper costs. Most people are also familiar with them. The study therefore adopted the questionnaire as a data collection technique. In the questionnaire questions are asked about people's gender, beliefs, opinions, characteristics and behaviour, and was then used as a source of data. The data collected described the variable of gender as a factor that influences information seeking amongst the students. The questions were categorised as follows:

- Preference questions which focused on the gender of the participants and what they thought about a library service.

- Reported behaviour and likely behaviour focusing on what the different genders say they do in terms of making choices to use or avoid using the library.

\section{Data analysis techniques}

The data collected was analysed quantitatively through Statistical Software for Social Sciences (SPSS) v 21.0 (IBM Corp., 2012, Armonk, New York). The following steps were taken:

- Organise and learn the data.

- Summarise the findings using averages, and present in the form of tables.

- A correlation analysis was done to examine how closely the variables were connected. These variables were the ones identified to have some bearing on information seeking. Correlation research helped in understanding the relationships between gender and the factors which were being considered in this study as the ones which were likely to drive information seeking amongst graduate students. The correlation coefficient ranges from +1 showing a perfect positive correlation and a -1 showing a negative correlation.

- The connections between variables were explored through discovering associations of two variables to a significant level using chi-square as a statistical test. It was tested as $P<0.05$ ( 1 in every 20). The significance level measures how frequently the conclusion will be wrong in the long run. A 0.05 or $5 \%$ significance level means that, in the long run, this type of conclusion will be wrong 5\% of the time (Mahuli \& Mahuli 2015).

The study also used multiple regression analysis to interpret the results. This allowed for exploration of interrelationship amongst the variables.

\section{Logistical and ethical considerations}

The purpose of ethical considerations in research is to ensure that no individual suffers from harmful effects as a consequence of research activities (Cooper 2003). Consequently, in this study great care was taken with the issues that could arise in relation to research and participants. This included harm, confidentiality, informed consent, and invasion of privacy.

\section{Findings, interpretation and discussion}

The purpose of this study was to investigate gender and cognitive factors the information-seeking seeking of graduate students at Kenyatta University Library. The study was based on the expectancy theory of motivation developed by Vroom (1964) and improved by Porter and Lawler (1968). The theory deals with the mental models/processes an individual undergoes to make choices - in this case, to use or avoid using the library reference service. The findings are discussed and interpreted below.

\section{General and demographic information}

This section presents data on personal characteristics in the study. These were based on gender. The respondents for this study were postgraduate students at Kenyatta University main campus library. The data were collected in November and December 2017. This enabled the study to access both regular, Institutional Based Programmes and Kenyatta University Digital School of Virtual and Open Learning students. Students participated out of an expected sample size of 373 . However, four responses were struck from the study during data cleaning, thus a total of 369 were analysed. Of these, $222(60.1 \%)$ were men and 147 were women (39.9\%). Table 1 shows the demographics of the study.

\section{Relationship between cognitive factors and gender}

\section{Outcomes and gender}

Outcome in this study may be explained as 'information needs satisfaction'. It had four main elements, as follows:

- Helpfulness of the service given by librarians.

- Satisfaction with information acquired through face-toface reference service.

TABLE 1: Demographic information-Gender.

\begin{tabular}{lc}
\hline Gender of the participants & Frequency \\
\hline Male & 222 \\
Female & 147 \\
\hline Total & 369 \\
\hline
\end{tabular}


- Usefulness of information from reference librarians.

- Satisfaction acquired through digital reference service.

Nearly three-quarters of the male students (72.6\%) reported that librarians gave helpful information, and an almost equal proportion (71.2\%) of female students felt that the information given to them by the face-to-face reference service was helpful. There seemed to be no difference between male and female students on the helpfulness of information given to them. A correlation coefficient of -0.003 was found for the relationship between gender and helpfulness of information given to them. The two variables related negatively. The score on the issue of satisfaction did not show significant differences amongst men and women, as shown in Table 2. A very small number was dissatisfied with the information acquired through the face-to-face reference service, as is shown by those who disagreed with the statement. About one-quarter $(24.0 \%)$ of the female students were not decided on whether they were satisfied or not, whereas $20 \%$ of their male counterparts were also undecided.

\section{Satisfaction and gender}

A total of $64.5 \%$ of the men and $56.4 \%$ of the women agreed that the information given to them by reference librarians was useful. Further analysis showed that less than one-fifth of both the women (19.3\%) and men (15.7\%) strongly disagreed that the information given by face-to-face reference librarians was useful (Table 2).

\section{Satisfaction with information acquired through digital reference}

There was a difference of opinion between genders on the satisfaction with information acquired though digital reference. The men (62.0\%) seemed to be more satisfied than the women (50.3\%) with the information acquired through digital means. There seemed to be no significant difference of opinion amongst the male and female students on an indicator of satisfaction with regard to information acquired

TABLE 2: Gender and satisfaction level.

\begin{tabular}{lcc}
\hline Gender & Satisfaction (\%) & Dissatisfaction (\%) \\
\hline Male & 64.5 & 15.7 \\
Female & 56.7 & 19.3 \\
\hline
\end{tabular}

through the digital reference service. A correlation of -0.84 was established between gender and outcomes.

\section{Gender and value of outcome}

On assessing whether there were gender differences on the cognitive variable of 'value of outcomes', three issues were considered:

- Desirability of information needed to be met through a face-to-face reference service,

- Importance for information needs to be met through a face-to-face reference service, and

- Value of information needs to be met through a face-toface reference service.

Table 3 shows the importance of information needs to be met through face-to-face reference services.

The majority of both male (63.3\%) and female (68.4\%) students agreed that it was very valuable for their information needs to be met through a face-to-face reference service. Those who felt that it was not valuable for their information needs to be met in this way were $16.5 \%$ of the women and $18.4 \%$ of the men. There did not seem to be significant differences between the gender perception of the value of the outcome variable (Table 3).

A similar finding was obtained on the importance for their information needs to be met through a face-to-face reference service. The majority of the participants $(62.6 \%$ male and $68.0 \%$ female) felt that the service was important, $17.9 \%$ of men and 19\% women disagreed, and 19.6 men and $16 \%$ women were neutral. On whether it was desirable for their information needs to be met through a face-to-face reference service, $67.6 \%$ of the male and 70.3 of the female students agreed with the statement that it was important for them to have their information needs met through such a service, whilst $14.5 \%$ of both male and female participants disagreed with the statement (Table 4).

When considering gender and value of outcome, there seemed to be no gender difference on the issues of desirability $(68.7 \%)$, importance $(64.7 \%)$ and value $(65.85 \%)$ of needs to

TABLE 3: Gender and value for information needs to be met through a face-to-face reference service.

\begin{tabular}{|c|c|c|c|c|c|c|c|c|c|c|c|c|}
\hline \multirow{3}{*}{$\begin{array}{l}\text { Gender of } \\
\text { respondent }\end{array}$} & \multicolumn{10}{|c|}{ High value for information met through reference service } & \multirow{2}{*}{\multicolumn{2}{|c|}{ Total }} \\
\hline & \multicolumn{2}{|c|}{ Strongly disagree } & \multicolumn{2}{|c|}{ Disagree } & \multicolumn{2}{|c|}{ Neutral } & \multicolumn{2}{|c|}{ Agree } & \multicolumn{2}{|c|}{ Strongly agree } & & \\
\hline & Frequency & $\%$ & Frequency & $\%$ & Frequency & $\%$ & Frequency & $\%$ & Frequency & $\%$ & Frequency & $\%$ \\
\hline Male & 17 & 7.9 & 23 & 10.5 & 40 & 18.3 & 84 & 38 & 56 & 25.3 & 220 & 100 \\
\hline Female & 12 & 7.9 & 13 & 8.6 & 22 & 15.1 & 59 & 40.1 & 41 & 28.3 & 147 & 100 \\
\hline Total & 29 & 7.9 & 36 & 9.8 & 62 & 17.1 & 143 & 38.8 & 97 & 26.5 & 367 & 100 \\
\hline
\end{tabular}

TABLE 4: Gender and importance of information needs to be met through a face-to-face reference service.

\begin{tabular}{|c|c|c|c|c|c|c|c|c|c|c|c|c|}
\hline \multirow{3}{*}{$\begin{array}{l}\text { Gender of } \\
\text { respondent }\end{array}$} & \multicolumn{10}{|c|}{ High importance for information needs met through reference service } & \multicolumn{2}{|l|}{ Total } \\
\hline & \multicolumn{2}{|c|}{ Strongly disagree } & \multicolumn{2}{|c|}{ Disagree } & \multicolumn{2}{|c|}{ Neutral } & \multicolumn{2}{|c|}{ Agree } & \multicolumn{2}{|c|}{ Strongly agree } & \multirow[b]{2}{*}{ Frequency } & \multirow[b]{2}{*}{$\%$} \\
\hline & Frequency & $\%$ & Frequency & $\%$ & Frequency & $\%$ & Frequency & $\%$ & Frequency & $\%$ & & \\
\hline Male & 13 & 5.8 & 27 & 12.1 & 43 & 19.6 & 97 & 43.8 & 41 & 18.8 & 221 & 100 \\
\hline Female & 9 & 6 & 15 & 10 & 24 & 16 & 72 & 49.3 & 28 & 18.7 & 148 & 100 \\
\hline Total & 22 & 5.9 & 42 & 11.4 & 67 & 18.2 & 169 & 46 & 69 & 18.7 & 369 & 100 \\
\hline
\end{tabular}


TABLE 5: Gender and willingness of face-to-face reference service personnel to help.

\begin{tabular}{|c|c|c|c|c|c|c|c|c|c|c|c|c|}
\hline \multirow{3}{*}{$\begin{array}{l}\text { Gender of } \\
\text { respondent }\end{array}$} & \multicolumn{10}{|c|}{ Rating regarding willingness of face-to-face reference service personnel to help } & \multicolumn{2}{|l|}{ Total } \\
\hline & \multicolumn{2}{|c|}{ Very low } & \multicolumn{2}{|l|}{ Low } & \multicolumn{2}{|c|}{ Neutral } & \multicolumn{2}{|c|}{ High } & \multicolumn{2}{|c|}{ Very high } & & \\
\hline & Frequency & $\%$ & Frequency & $\%$ & Frequency & $\%$ & Frequency & $\%$ & Frequency & $\%$ & Frequency & $\%$ \\
\hline Male & 18 & 8.3 & 34 & 15.4 & 51 & 23.2 & 96 & 44.3 & 19 & 8.8 & 218 & 100 \\
\hline Female & 22 & 15.1 & 34 & 23 & 37 & 25.7 & 42 & 28.9 & 11 & 7.2 & 146 & 100 \\
\hline Total & 40 & 11.1 & 68 & 18.4 & 88 & 24.2 & 138 & 38.2 & 30 & 8.2 & 364 & 100 \\
\hline
\end{tabular}

TABLE 6: Courteousness of face-to face-reference service personnel

\begin{tabular}{lcccccc}
\hline Gender & \multicolumn{5}{c}{ Rating regarding courteousness of face-to-face reference } \\
& \multicolumn{7}{c}{ service personnel } \\
\cline { 2 - 7 } & Low (\%) & Very low (\%) & Total (\%) & High (\%) & Very high (\%) & Total (\%) \\
\hline Male & 15.4 & 8.3 & 23.7 & 39.7 & 10.5 & 50.2 \\
Female & 23.0 & 15.1 & 38.1 & 29.5 & 9.4 & 38.9 \\
\hline
\end{tabular}

be met through a face-to-face reference service. The gender variable showed a -0.52 correlation to value of outcome. This was a medium negative correlation between the two variables, and was considered significant at a 0.05 level of significance.

There were comparatively few participants who did not think it was valuable, important and desirable for the information needs to be met through face-to-face interaction.

\section{Gender and interaction service quality}

Interaction service quality was assessed in two ways:

- Willingness of face-to-face personnel to help.

- Courteousness of face-to-face reference service personnel.

Male respondents thought that the service personnel had either a high $(44.3 \%)$ or very high $(8.8 \%)$ willingness to help - this represented over half $(53.1 \%)$ of the male students. The female respondents who rated the service personnel's willingness to help as either high $(28.9 \%)$ or very high $(7.2 \%)$ represented $36.1 \%$. There was a $17.0 \%$ difference of rating between male and female students in this regard. Of the male respondents, $15.4 \%$ thought that the service personnel had a low willingness to help, whilst $8.3 \%$ rated this as very low. Female respondents, however, rated these as low (23\%) or very low (15.1\%). The situation was as shown in Table 5.

The male respondents rated the service personnel's courtesy to be either high $(39.7 \%)$ or very high $(10.5 \%)$. This meant that about half $(50.2 \%)$ of the male respondents rated the service personnel's courtesy positively. By comparison, only $9.5 \%$ of the female students rated the service personnel's courtesy as high $(29.5 \%)$ or very high $(9.4 \%)$ (Table 6$)$.

\section{Courtesy and gender}

Almost half of the female students (38.9\%) rated courteousness as being high or very high. Overall, one-third (30.9\%) of the female respondents considered the service personnel's courteousness to be low or very low, whereas only one-fifth $(21.0 \%)$ of the male respondents had the same rating. Despite the differences between male and female ratings, gender did not seem to be a significant enough discriminator for this metric. Overall interaction service quality and gender

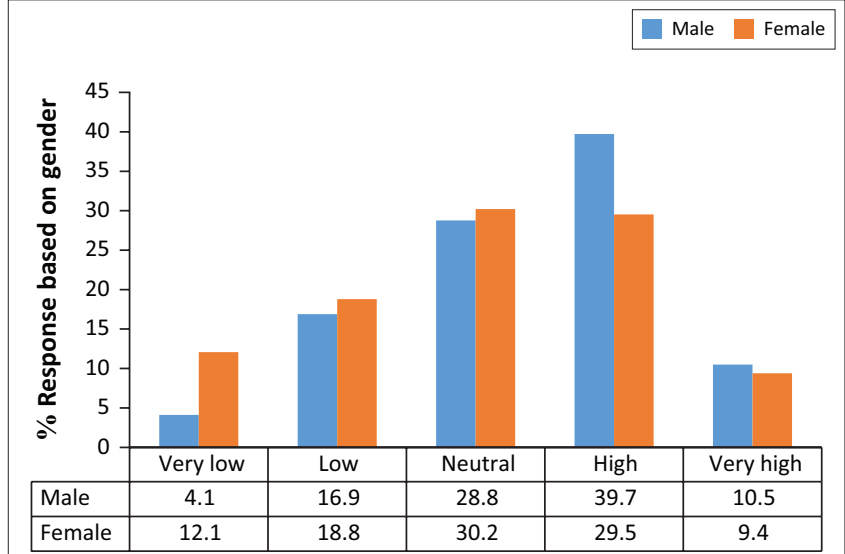

Rating: Courteousness of face-to-face reference service personnel

FIGURE 2: Courteousness of face-to-face service personnel.

showed a negative relationship (-0.156), meaning that gender and interaction service quality were negatively correlated. Figure 2 shows this relationship.

\section{Gender and overall satisfaction with a face-to- face reference service}

The satisfaction ratings show that the female students were less satisfied than male students. A correlation of -0.100 was found between overall satisfaction and gender. This meant that gender and overall satisfaction were negatively correlated. Equally, on the likelihood of using a face-to-face reference service in the future, more male students $(68.6 \%)$ were likely to do so than female students $(61.6 \%)$.

Only $20.3 \%$ of male and $29.2 \%$ of female students were not likely or very unlikely to recommend the face-to-face reference service to friends and colleagues. Overall library user satisfaction with the service was negatively correlated with gender, indicating that the decisions of recommending or not recommending face-to-face reference service to friends and colleagues were not likely to be influenced by gender. The ratings for the likelihood to use the service in the future and the likelihood of recommending the service to friends and colleagues were comparatively close amongst male and female students. They were both likely to use the service and to recommend the service to friends. Table 7 shows the likelihood of using a face-to-face reference service in the future.

\section{Gender and effort by users of face-to-face reference service}

Effort by the user of the reference service was considered in the following ways: 
TABLE 7: Gender and likelihood of using a face-to-face service in future.

\begin{tabular}{|c|c|c|c|c|c|c|c|c|c|c|c|c|}
\hline \multirow{3}{*}{$\begin{array}{l}\text { Gender of } \\
\text { respondent }\end{array}$} & \multicolumn{10}{|c|}{ Likelihood of use of face-to-face reference service in future } & \multicolumn{2}{|l|}{ Total } \\
\hline & \multicolumn{2}{|c|}{ Very unlikely } & \multicolumn{2}{|c|}{ Unlikely } & \multicolumn{2}{|c|}{ Neutral } & \multicolumn{2}{|c|}{ Likely } & \multicolumn{2}{|c|}{ Very likely } & & \\
\hline & Frequency & $\%$ & Frequency & $\%$ & Frequency & $\%$ & Frequency & $\%$ & Frequency & $\%$ & Frequency & $\%$ \\
\hline Male & 16 & 7.5 & 28 & 12.8 & 24 & 11.1 & 137 & 62.4 & 14 & 6.2 & 219 & 100 \\
\hline Female & 11 & 7.3 & 32 & 21.9 & 14 & 9.3 & 84 & 58.3 & 5 & 3.3 & 146 & 100 \\
\hline Total & 27 & 7.4 & 60 & 16.4 & 38 & 10.3 & 221 & 60.7 & 19 & 5 & 365 & 100 \\
\hline
\end{tabular}

TABLE 8: Gender and effort by users of face-to-face reference service.

\begin{tabular}{|c|c|c|c|c|c|c|c|c|c|c|c|c|}
\hline \multirow{3}{*}{$\begin{array}{l}\text { Gender of } \\
\text { respondent }\end{array}$} & \multicolumn{10}{|c|}{ Extent of use of face-to-face reference service in future } & \multicolumn{2}{|l|}{ Total } \\
\hline & \multicolumn{2}{|c|}{ Very low } & \multicolumn{2}{|l|}{ Low } & \multicolumn{2}{|c|}{ Medium } & \multicolumn{2}{|c|}{ High } & \multicolumn{2}{|c|}{ Very high } & & \\
\hline & Frequency & $\%$ & Frequency & $\%$ & Frequency & $\%$ & Frequency & $\%$ & Frequency & $\%$ & Frequency & $\%$ \\
\hline Male & 15.69 & 7.1 & 47.29 & 21.3 & 107.22 & 48.3 & 44.4 & 20.0 & 9.55 & 4.3 & 222 & 100.0 \\
\hline Female & 6.9 & 4.7 & 40.57 & 27.6 & 66.73 & 45.4 & 24.1 & 16.4 & 8.67 & 5.9 & 147 & 100.0 \\
\hline Total & 22.59 & 11.8 & 87.86 & 48.9 & 173.95 & 93.7 & 68.5 & 36.4 & 18.22 & 10.2 & 369 & 100.0 \\
\hline
\end{tabular}

- Extent of use of reference service.

- Level of effort spent using face-to-face reference service.

The scores between male and female respondents on the extent of use of face-to-face reference service were close. The majority $(69.6 \%)$ of the male respondents considered the extent of use to be either low $(21.3 \%)$ or medium $(48.3 \%)$. The female repondents $(73.0 \%)$ assessed the extent of their effort to be either low (27.6\%) or medium (45.4\%) as well. Both male and female participants scored the extent of use of face-to-face reference to be either low or medium. Onefifth of the men assessed their extent of effort to be high $(20.0 \%)$, and $16.4 \%$ of women felt the same. A small proportion $(4.3 \%$ of the male respondents and $5.9 \%$ of the females) rated their effort to be very high. There seemed to be an insignificant difference between men and women (Table 8).

There was a negative correlation $(-0.054)$ between gender and the extent of use of face-to-face reference service.

The level of effort spent using face-to-face reference service was reported to be mainly low (24.0\%) and medium (48.8\%) for male respondents, whilst the female respondents had a combined level of $70.4 \%$ clustered around low and medium effort. Both men (72.8\%) and women (70.4\%) had very similar ratings for their level of effort.

On whether there was a difference between genders, the extent of use of face-to-face reference service was close. The majority of men $(69.6 \%)$ considered the extent of use to be either low $(21.3 \%)$ or medium $(48.3 \%)$. The women $(73.0 \%)$ assessed the extent of their effort to be either low $(27.6 \%)$ or medium (45.4\%). Both genders assessed the extent of use of a face-to-face reference service to be either low or medium. One-fifth of the men assessed their extent of effort to be high $(20.0 \%)$, versus $16.4 \%$ of the women. A small proportion rated their effort to be very high $(4.3 \%$ of the male students and $5.9 \%$ of the females). There seemed to be an insignificant difference between male and female respondents. On the correlation between gender and effort, there was a negative correlation $(r=0.54)$. A medium negative correlation was established.

\section{Performance and/or accomplishment of the service and gender}

Performance of the service was assessed in two ways:

- Problems faced with digital reference service.

- Problems faced communicating with reference librarian.

About $58.6 \%$ of male and $54.9 \%$ of female students reported that they had problems with the digital reference service. About $12.3 \%$ of the male respondents and $12.6 \%$ of the female were neutral. This should mean that over $60 \%$ of students of both genders had problems with the digital reference service. Only about one-third (28.9\%) of the men and a similar proportion of the women $(32.5 \%)$ reported that they did not have problems with the digital reference service. Performance/accomplishment of service by users was found to be negatively correlated to gender $(-0.47)$.

On the problems faced with communicating with the reference librarian, $49.4 \%$ of the men and $40.8 \%$ of the women reported that they did not have problems. At the same time one-third, $31.6 \%$ of men and $34.2 \%$ of women, acknowledged that they had problems with communication with the reference librarians. Hence, about half of the respondents (both male and female) could communicate with the reference librarians without problems. Both female and male respondents had very close findings on the variable of communicating with reference librarians, and a negative correlation coefficient of -0.02 was found between gender and ability to communicate with the reference librarian.

\section{Users' past experience and gender}

Users' past experience was assessed in two main areas:

- Experience with reference service.

- Experience with digital reference service.

Users' experience with the face-to-face reference service was rated to be mainly low (27.6\%) and medium (44.2\%). This meant that $71.8 \%$ of the respondents rated their experience to be mainly medium and low. Only about $8.9 \%$ were neutral. About one fifth (19.2\%) reported that they had a high or very high rating of their experience with the face-to-face reference 
service. These rating scores were comparatively close for both male $(70.7 \%)$ and female $(73.5 \%)$ respondents. A similar pattern was found in those respondents who considered their experience to be high $(19.7 \%$ for male students and $18.6 \%$ for females). Both male and female respondents scored their experience similarly on the face-to-face reference service.

A negative correlation coefficient of -0.010 was found. Gender and level of experience were negatively correlated. The level of experience with digital reference service was mainly low (20.7\%) and medium (33.1\%). This meant that about half of the men (54.2\%) and women (53.3\%) had a low or medium experience. Apparently, $37.6 \%$ of men and $37.5 \%$ of the women considered their experience with the digital service to be high. An analysis of the level of experience in both male and female respondents showed that it was close and there was little difference. A negative correlation of -0.026 was found between level of experience with digital and gender.

\section{Cognitive factors and gender}

Previous studies had found two main positions on gender and user services. Some found that there was a difference between men and women on the use of services (Brindesi et al. 2013; Ford, Miller \& Moss 2001; Halder et al. 2010). Some studies (Brown 2001; Hsieh \& Wu 2015) found no gender difference amongst students' use of a digital library (Rehman 2016). This study found no gender differences in all the variables considered, including interaction service quality, outcome (need satisfaction), service satisfaction, users' performance of service, past experience, expectancy and effort. The implication of this was that there is no need for mainstreaming gender in service programming in the library service.

\section{Conclusion}

This study examined gender as one of the drivers and triggers of cognitive factors that influence information-seeking behaviour, prior to contacting the librarian. Cognitive information-seeking behaviour was considered as an approach to how an individual applies his or her mental models of the world to process information seeking. The focus was user-covered and the study utilised the expectancy theory of motivation to explain information seeking. The expectancy theory can explain information seeking as being driven by cognitive factors. These cognitive factors that, combined together, influence information-seeking process prior to making contact with reference librarians, include interaction service quality, outcomes (needs satisfaction), overall satisfaction of service, users' competencies in performing a service, expectancies and past experience. Consequently, this study investigated whether there was a relationship between gender and these cognitive factors that influences information seeking. The study found that there was no relationship between gender and all the cognitive variables. Information seeking was not affected by gender as there was no gender-based difference. There were more similarities between male and female respondents on cognitive information seeking, however, gender made no difference in predicting cognitive information seeking.

\section{Implication}

The implication of this finding was that the library need not develop any interviewing strategies to develop mental images or experiences based on gender.

\section{Recommendations}

An appropriate information literacy programme needs to be developed which would integrate information-seeking skills in both learning and teaching, aimed at developing positive mental experiences. The library system developer would want to make systems as convenient and friendly as possible. Such a user-centred system should recognise that students of either gender search for information as individuals, and should help them to build high self-esteem.

Equally, future studies could investigate the relationship between gender and cognitive factors that influence information seeking in different settings, for the purpose of drawing clearer and wider generalisations.

\section{Acknowledgements}

This study would not have been possible without the exceptional support of Professor Jing Chen, and the whole community of Central China Normal University for agreeing to sponsor the study. We forever remain grateful.

\section{Competing interests}

The authors have declared that no competing interest exists.

\section{Authors' contributions}

All authors contributed equally to this work.

\section{Ethical consideration}

This article followed all ethical standards as set out by the scientific community of researchers.

\section{Funding information}

The publication cost is funded by the Central China Normal University.

\section{Data availability statement}

Data sharing is not applicable to this article as no new data were created or analysed in this study.

\section{Disclaimer}

The views and opinions expressed in this article are those of the authors and do not necessarily reflect the official policy or position of any affiliated agency of the authors. 


\section{References}

Ary, D., Jacobs, L.C. \& Sorensen, C., 2010, 'Introduction to research in education', Wadworth 35, 63-68.

Bandura, A., 1977, 'Self-efficacy: Towards unifying theory of behavioural change', Psychological Review 84(2), 191-215. https://doi.org/10.1037/0033-295X.84. 2.191

Brindesi, H., Monopoli, M. \& Kapidakis, S., 2013, 'Information seeking and searching habits of Greek physicists and astronomers: A case study of undergraduate habits of Greek physicists and astronomers: A case study of undergraduate doi.org/10.1016/j.sbspro.2013.02.119

Brown, S.P., 2001, 'Self-efficacy as a moderator of information-seeking effectiveness', Journal of Applied Psychology 86(5), 1043-1051. https://doi.org/10.1037/0021 9010.86.5.1043

Campbell, J.J., Dunnette, M.D., Lawler, E.E. \& Weick, K.E., 1970, Managerial behavior; Performance and effectiveness, McGraw-Hill Book Company, New York, NY.

Cochrun, W.G., 1977, Sampling techniques, Wiley \& Sons, New York, NY.

Conrad, S., 2014, 'Toward improved discoverability of scholarly content: Cross-sector collaboration essentials', Collaborative Librarianship 6(1), 42-46.

Cooper, D.R.A.S., 2003, Business research methods, McGraw-Hill, Irwin, Boston.

Ford, N., Miller, D. \& Moss, N., 2001, 'The role of individual differences in internet searching: An empirical study', Journal of the American Society for Information Science and Technology 52(12), 1049-1066. https://doi.org/10.1002/asi.1165

Halder, S., Ray, A. \& Chakrabarty, P.K., 2010, 'Gender differences in information seeking behaviour in three universities in West Bengal, India', The Internationa Information \& Library Review 42, 242-251. https://doi.org/10.1080/10572317.20 10.10762869

Hsieh, Y.-Y. \& Wu, K.-C., 2015, 'The influence of gender difference on the information seeking behaviors for the graphical interface of children's digital library', Universa Journal of Educational Research 3(3), 200-206. https://doi.org/10.13189/ ujer.2015.030305

Kassim, N.A., 2017, 'Evaluating users' satisfaction on academic library performance', Malaysian Journal of Library \& Information Science 14, 101-115.
Kenyatta University, 2013, Historical development of Kenyatta University Library, Kenyatta University Press, Nairobi, Kenya.

Kenyatta University, 2018, Postgraduate Prospectus, Kenyatta University Press, Nairobi, Kenya.

Khaola, P.M. \& Mabilikoane, M., 2015, 'Perception of library service quality, satisfaction and frequency of use of library resources', Journal of Humanities and social sciences 7, 44-52.

Mahuli, A.V. \& Mahuli, S.A.,2015, Significance of statistical significance. Journal of Dental Research and Review 2(3), 106.

Masinde, J.M., 2016, 'Investigation of mobile library user needs of international students based on the expectancy theory of motivation', Research Journal of Library Sciences 4, 14-30.

Nardi, P.M., 2015, Doing survey research: A guide to quantitative research, Routledge, London, United Kingdom.

Porter, L.W. \& Lawler, E.E., 1968, Managerial attitudes and performance, Dorsey Press, Illinois.

Rehman, S.U., 2016, 'Measuring service quality in public and private sector university libraries of Pakistan', Pakistan Journal of Information Management \& Libraries 13(1), 1-11.

Sacks, M., 1994, Cognitive closure and the limits of understanding, Wiley Online Library, London, United Kingdom

Saunders, M., Lewis, P. \& Thornhill, A., 2009, Research methods for business students, Pearson, New York, NY.

Savolainen, R., 2013, 'Approaching the motivators for information seeking: Viewpoint of attribution theories', Library and Information Science research and International Journal 35(1), 63-68. https://doi.org/10.1016/j.lisr.2012.07.004

Vroom, V.H., 1964, Work and motivation, Wiley.

Wisdom, J. \& Creswell, J.W., 2013, Mixed methods: Integrating quantitative and qualitative data collection and analysis while studying patient-centered medical home models, Agency for Healthcare Research and Quality, Rockville, MD.

Wu, K.C., Tang, Y.M. \& Tsai, C.Y., 2014, 'Graphical interface design for children seeking information in a digital library', Visualization in Engineering 2(5). https://doi. org/10.1186/2213-7459-2-5 